\title{
Editorial to selected papers from the 15th IMEKO TC10 Workshop on Technical Diagnostics - "Technical Diagnostics in Cyber-Physical Era"
}

\author{
Lorenzo Ciani, Marcantonio Catelani \\ Department of Information Engineering, University of Florence, Italy
}

Section: EDITORIAL

Citation: Lorenzo Ciani, Marcantonio Catelani, Editorial to selected papers from the 15th IMEKO TC10 Workshop on Technical Diagnostics - "Technical Diagnostics in Cyber-Physical Era", Acta IMEKO, vol. 7, no. 1, article 2, March 2018, identifier: IMEKO-ACTA-07 (2018)-01-2

Editor: Dušan Agrež, University of LUUBLANA, Slovenia

Received March 23, 2018; In final form March 24, 2018; Published March 2018

Copyright: @ 2018 IMEKO. This is an open-access article distributed under the terms of the Creative Commons Attribution 3.0 License, which permits unrestricted use, distribution, and reproduction in any medium, provided the original author and source are credited

Corresponding author: Lorenzo Ciani, email: lorenzo.ciani@unifi.it

Dear Reader,

A Section of this Issue is dedicated to eight papers that were originally presented at 15th IMEKO TC10 Workshop on Technical Diagnostics in Budapest (Hungary) and that are here presented in their extended versions.

Technical diagnostics is taking in the years an increasingly important role. Behind this, the fact that in high-tech industry and in different fields of application it is mandatory to fulfil the requirements related to diagnostics, reliability, maintainability and logistic support as well as risk and safety assessment. The capability to monitor and diagnose a component, a system, an equipment or an industrial plant - in general term, an item with the aim to verify its functions represents the starting point for more complex RAMS (Reliability, Availability, Maintainability and Safety) evaluations and assessment.

The 15th IMEKO TC10 Workshop was held at the Danubius Hotel Gellért in Budapest, Hungary, on June 6-7, 2017. This Workshop aims to create a forum for advancing knowledge and exchange ideas on methods, principles, instruments and tools, standards and industrial applications on Technical Diagnostics as well as their diffusion across the scientific community. Participants have an excellent opportunity to meet top specialists from industry and academia all over the world and to enhance their international cooperation. The program will feature industry leading keynote speakers and selected presentations.

The first paper by Yi Huang and Clemens Gühmann proposes a method to estimate the temperatures of the stator winding, the rotor cage and the stator core of an asynchronous machine using Kalman filter. A 4th-order Kalman filter was implemented in Matlab and both the simulation experiment and the test bench experiment are performed well under S1 and S6 condition.

The second paper by Daniel Peters, Patrick Scholz and Florian Thiel analyses secure software constructions for general purpose operating systems, i.e., Linux and Windows. The authors look at measuring instruments under legal control, which have become powerful devices running under such operating systems. The frameworks presented here, are constructed to fulfil the requirements of legal metrology such as the Measuring Instruments Directive MID 2014/32/EU and the WELMEC 7.2 Software Guide. The main goal is to make current measuring instruments more resistant against software vulnerabilities and attacks from open networks like the Internet.

The third paper by Gábor Kohlrusz, Krisztián Enisz, Bence Csomós and Dénes Fodor investigates and discusses the input and output capacitors of a digitally controlled Buck converter have been tested in 4 different cases with unused and worn out capacitors. A complex simulation environment has been implemented where digital and analogue domains can be connected. The presented diagnostic approach aims to analyse signals that are used by the control loop in order to avoid expensive additions to existing circuits.

The fourth paper in this issue is authored by Balázs Scherer introduces a novel approach, and the first part of a toolset for non-intrusive diagnostics of cyber-physical systems. The main goal is to create a toolset, which supports multiple nonintrusive diagnostic interface options for testing cyber-physical systems. The sample implementation is currently a basic version enabling only few functionalities of the XCP protocol, but it is 
suitable to evaluate the concept. First measurements shown that the concept is good, and this method enables the diagnostic of low cost, resource frugal cyber-physical systems with a relatively good data bandwidth.

The fifth contribution by Dario Pasadas, Artur Lopes Ribeiro and Helena Geirinhas Ramos reports a study concerning the penetration of eddy currents in metallic nonferromagnetic materials in the presence of linear sub-surface cracks. Simulations were performed for a set of different lengths and depths of the crack using a sinusoidal excitation current with uniform magnetic field distribution.

The sixth paper by Piotr Bilski and Bartosz Polok investigates and discusses the optimization of various RBF network-based classifier in the diagnostics of analogue circuits. Multiple parameters, including the width of the activation function, number of neurons in the hidden layer and coding scheme in the output layer were verified. The testability of the circuit was also analysed.

The seventh paper is the result of an international collaboration among researchers from Tecnalia Research and Innovation, Industry and Transport Division, Miñano (Araba), Spain, the Division of Operation and Maintenance Engineering, Luleå University of Technology, Sweden and the Department of Information Engineering, University of Florence, Italy. This paper reviews the challenges of maintaining the linear assets, focusing on inspections. It also provides a conceptual framework for the use of autonomous inspection and maintenance practices for linear assets to reduce maintenance costs, human involvement, etc., whilst improving the availability of linear assets by effective use of autonomous robots and data from different sources.

Finally, the eight paper in this issue is authored by Timotei Erdei, Zsolt Molnár, Nwachukwu C. Obinna and Géza Husi. This paper proposes a design of an augmented reality based navigation system, as well as investigate its potential areas of application within the industry. With the ascent of Industry 4.0 (IoT), systems such as Augmented Reality and Virtual Reality benefit from the availability of bulk sensory data.

It was a great honour for us to act as Guest Editors for this issue of ACTA IMEKO, both from the perspective of working for a high-profile scientific journal and also, of promoting TC10's great tradition workshop, now reaching the 15th edition.

Let us also take this occasion to remind everyone of the next XXII World Congress of the International Measurement Confederation (IMEKO) that will be held in Belfast, UK, on September 3rd6th, 2018 and we are pleased to invite you to attend the conference.

\section{Lorenzo Ciani}

Guest Editor

Marcantonio Catelani

IMEKO TC10, Chair 